\title{
Identification of transcription factors potential related to brown planthopper resistance in rice via microarray expression profiling
}

\author{
Yubing Wang ${ }^{1,2}$, Huimin Guo ${ }^{1,2}$, Haichao Li ${ }^{1}$, Hao Zhang ${ }^{1,2}$ and Xuexia Miao ${ }^{1 *}$
}

\begin{abstract}
Background: Brown planthopper (BPH), Nilaparvata lugens Stål, is one of the most destructive insect pests of rice. The molecular responses of plants to sucking insects resemble responses to pathogen infection. However, the molecular mechanism of BPH-resistance in rice remains unclear. Transcription factors (TF) are up-stream regulators of various genes that bind to specific DNA sequences, thereby controlling the transcription from DNA to mRNA. They are key regulators for transcriptional expression in biological processes, and are probably involved in the $\mathrm{BPH}$-induced pathways in resistant rice varieties.
\end{abstract}

Results: We conducted a microarray experiment to analyze TF genes related to BPH resistance in a Sri Lankan rice cultivar, Rathu Heenati (RHT). We compared the expression profiles of TF genes in RHT with those of the susceptible rice cultivar Taichun Native 1 (TN1). We detected 2038 TF genes showing differential expression signals between the two rice varieties. Of these, 442 TF genes were probably related to BPH-induced resistance in RHT and TN1, and 229 may be related to constitutive resistance only in RHT. These genes showed a fold change (FC) of more than 2.0 $(P<0.05)$. Among the $442 \mathrm{TF}$ genes related to $\mathrm{BPH}$-induced resistance, most of them were readily induced in TN1 than in RHT by BPH feeding, for instance, 154 TF genes were up-regulated in TN1, but only 31 TF genes were up-regulated in RHT at 24 hours after BPH infestation; 2-4 times more TF genes were induced in TN1 than in RHT by BPH. At an FC threshold of $>10$, there were 37 induced TF genes and 26 constitutive resistance TF genes. Of these, 13 were probably involved in BPH-induced resistance, and 8 in constitutive resistance to BPH in RHT.

Conclusions: We explored the molecular mechanism of resistance to BPH in rice by comparing expressions of TF genes between RHT and TN1. We speculate that the level of gene repression, especially for early TF genes, plays an important role in the defense response. The fundamental point of the resistance strategy is that plants protect themselves by reducing their metabolic level to inhibit feeding by BPH and prevent damage from water and nutrient loss. We have selected $21 \mathrm{TF}$ genes related to BPH resistance for further analyses to understand the molecular responses to BPH feeding in rice.

Keywords: Transcription factor, Oryza sativa L, Nilaparvata lugens Stål, Microarray

\section{Background}

Rice, Oryza sativa L., is the staple food of more than three billion people in Asia. In natural environments, rice plants are often attacked by microbial pathogens and insect pests. One of the most destructive insect pests of rice is the brown planthopper (BPH), Nilaparvata lugens Stål. BPH

\footnotetext{
* Correspondence: xxm@sibs.ac.cn

${ }^{1}$ Key Laboratory of Insect Developmental and Evolutionary Biology, Institute of Plant Physiology and Ecology, Shanghai Institutes for Biological Sciences, Chinese Academy of Sciences, Shanghai 200032, P.R. China

Full list of author information is available at the end of the article
}

use their stylets to probe intercellularly through epidermal and mesophyll cell layers until they reach the phloem sieve element to extract phloem sap as their food [1]. This differs from the feeding pattern of chewing insects. Their feeding activity extracts the phloem sap of rice and causes damage known as 'hopper burn', which can be lethal to rice plants attacked by large populations of the insect. Their feeding also transmits certain rice viruses such as ragged stunt virus and grassy stunt virus [2]. In recent years, BPH infestations have devastated many rice crops in Asia [3]. Therefore, the rice resistance against $\mathrm{BPH}$ is very important to rice 
production. Generally, according to the gene's expression level whether can be affected by $\mathrm{BPH}$ attack, the rice resistance were divided into induced resistance and constitutive resistance. The "induced resistance" refers to induced changes in preference, performance, or reproductive success of the attacker [4]. Most of plants will produce induced resistance reaction once they were attacked by herbivore insects. While in contrast, constitutive plant resistance refers to the constant level of resistance in a plant, regardless of herbivore attack. Most prominent resistance factor, for example the morphological features and the chemical composition of the plant, have been recognized as constitutive resistance characters [5].

Few studies have been conducted on the molecular responses of plants to sucking insects. Most studies on plant defense responses to phloem-feeding insects have focused on aphids and whiteflies [6,7]. In the interaction between rice and $\mathrm{BPH}$, gene expression is controlled after activation of salicylic acid (SA)-dependent and jasmonic acid (JA)/ ethylene (ET)-dependent signaling pathways $[8,9]$; this was thought to be a response to pathogen infection [10]. Analyses of physiological responses and gene expression profiles in rice showed that the genes significantly induced or repressed by $\mathrm{BPH}$ infestation were involved in several different pathways, including cellular transport, signal transduction, metabolism, macromolecular degradation, and plant defenses $[9,11,12]$. Although differential expression profiles related to $\mathrm{BPH}$ infestation have been analyzed by several groups, the molecular mechanism of $\mathrm{BPH}$ resistance in rice is remains unclear.

Previously, we conducted a series of microarray analyses to find potential BPH resistance genes in a Sri Lankan rice cultivar, Rathu Heenati (RHT). We compared its gene expression profiles under BPH stress with those of the susceptible cultivar Taichun Native 1 (TN1), which was used as the negative control [13]. Using this screening strategy, we identified many transcription factor (TF) genes related to $\mathrm{BPH}$ resistance. The products of these genes play important regulatory roles in many resistance-related pathways. TFs are proteins that can bind to specific DNA sequences, thereby controlling the transcription from DNA to mRNA $[14,15]$. They are key regulators of transcriptional expression in many biological processes [16]. Previous studies also indicated that TF genes directly or indirectly regulate the plant defense response [17-20]. In the model plant Arabidopsis, bHLH and WRKY gene families have been implicated in $R$ gene-mediated resistance against aphids [18]. The Arabidopsis TF AtWRKY70 modulates the crosstalk between the salicylic acid and jasmonic acid signaling pathways, which play important roles in plant defense against pathogens [21-23]. Over-expression of a rice TF gene, WRKY89, increased plant resistance to white-backed planthopper, a phloem-feeding insect [24]. OsERF3 is another rice TF gene that belongs to the AP2/ EREBP family. It positively regulates TrypPI activity and plays a role in the resistance of rice to the chewing herbivore, rice striped stem borer. It also appears to be a negative regulator of resistance against $\mathrm{BPH}$ [8]. TF genes that contain a zinc finger motif have been implicated in the regulation of plant tolerance to biotic or abiotic stresses [25-28]. Other TF families, including MYB $[29,30]$, NAC domain-containing [31-34], and bZIP families [35-38], also have important roles in plant-defense response pathways. DNA microarray technology provides a high-throughput method to measure expression levels of thousands of genes simultaneously. The technique is a powerful tool for global analysis of altered gene expression in plants under different conditions [39-41]. The results of previous studies suggest that it is feasible and practical to use microarrays to measure the expression levels of TF genes in rice plants infested by $\mathrm{BPH}$.

In this study, we analyzed the expression profiles of 2720 TF genes in an Affymetrix rice genome array at two time points during the plant-BPH interaction. Analyses of differential expression profiles between susceptible (TN1) and resistant (RHT) rice cultivars allowed us to identify TF genes that showed significant changes in expression levels after $\mathrm{BPH}$ infestation. We identified 37 induced and 26 constitutive TF genes related to $\mathrm{BPH}$ resistance. Of these, 21 TF genes were further analyzed and identified as those that were most likely to be related to $\mathrm{BPH}$ resistance in RHT. We speculate about the molecular mechanisms of resistance to BPH in RHT based on the expression patterns of TF genes after $\mathrm{BPH}$ feeding.

\section{Methods}

\section{Plant and insect materials}

We used the BPH-susceptible rice cultivar Taichung Native 1 (TN1) and the BPH-resistant cultivar Rathu Heenati (RHT) [42]. Pre-germinated seeds were sown in a plastic tray $(60 \times 45 \times 45 \mathrm{~cm})$ and were grown under a 14$\mathrm{h}$ light/10-h dark photoperiod at $28 / 22^{\circ} \mathrm{C}$. The TN1 cultivar was used to raise the $\mathrm{BPH}$ populations.

The $\mathrm{BPH}$ population was obtained from the China $\mathrm{Na}$ tional Rice Research Institute, Fuyang, Zhejiang. The $\mathrm{BPH}$ population was reared in a greenhouse under the same temperature and light regime described above. We used second or third instar nymphs of $\mathrm{BPH}$ for infestation experiments, and the fourth or fifth instar nymphs for the host-plant choice experiment.

\section{BPH infestation and sample collection}

The second or third instar nymphs were transferred to 6-week-old seedlings (10 $\mathrm{BPH}$ nymphs per plant) in a box covered with nylon mesh. Stems of the rice plant infected by $\mathrm{BPH}$ were collected as samples for 
microarray and $\mathrm{qPCR}$ at $0,2,4,8,12,18,24$, or $36 \mathrm{~h}$ after BPH infestation. The stems were transferred to liquid nitrogen immediately and then stored at $-70^{\circ} \mathrm{C}$.

The herbivore host-plant choice experiment was performed in plastic pots with two plants each. To determine the colonization preferences of $\mathrm{BPH}$, the two plants were covered with a plastic cage for each experiment (diameter $15 \mathrm{~cm}$, height $50 \mathrm{~cm}$ ), and 20 nymphs of 4 5 instar BPH were introduced into the cage. The BPH nymphs on each plant were counted at $2,4,6,8,10,12$, $24,36,48$, and $72 \mathrm{~h}$ after release. This experiment had five replicates.

\section{RNA extraction, Affymetrix microarray hybridization, and data normalization}

Total RNA was extracted using the QIAGEN RNA Extraction kit (QIAGEN, Hilden, Germany) according to the manufacturer's instructions. The RNA samples were purified with the QIAGEN RNAeasy kit (QIAGEN, Hilden, Germany), and an Agilent 2100 Bioanalyzer was used to monitor sample quality. Only RNA samples from the 0,8 , and $24 \mathrm{~h}$ time-points were used for Affymetrix microarray hybridizations.

The Shanghai Bio Corporation carried out Affymetrix rice genome microarray hybridizations. RNA target preparation and microarray hybridization were performed with the GeneChip ${ }^{\circledR}$ 3' IVT Express kit (Affymetrix, Cleveland, USA) and GeneChip ${ }^{\circledR}$ Hybridization, Wash, and Stain kit (Affymetrix, Cleveland, USA), strictly following the manufacturer's instructions. The signal intensity for each probe set on the GeneChip microarray was detected with a GeneChip ${ }^{\circledR}$ Scanner 3000 (Affymetrix, Cleveland, USA), and the raw signal value for each probe set was analyzed with GeneChip operating software (GCOS; Affymetrix, Cleveland, USA). The original data has already submitted to GEO, and the GEO record is "GSE29967 - Expression data from rice after brown planthopper attack".

For microarray analyses, we included six treatments of RHT and TN1 before and after BPH infestation with three biological repeats for each treatment. We conducted quintile normalization for all microarrays using MAS 5.0 to standardize the distribution of probe intensities for each array in a set of arrays. For quality control of samples, we compared all sample expression files using principal component analysis (PCA) to ensure that all samples representing the same experimental conditions were similar to each other. Primary screening was performed with Genespring GX 11.5 (Agilent Technologies, Santa Clara, CA) using one-way ANOVA and a Benjamini Hochberg false discovery rate threshold of less than 0.05. The expression values were compared pair-wise with the fold-change tool and a Student's t-test was performed during this step to obtain the P-value for each probe set.

\section{Transcription factor screening and microarray probe set filters}

The rice transcription factors analyzed in this experiment were described in the transcription factor database [43]. According to the annotation of Affymetrix genome microarray (release 30), we screened for TF genes that were differentially induced or repressed after BPH infection in RHT and TN1 with a fold change (FC) of $>2.0$ and a $P$-value of $<0.05$. The results were shown as a Venn diagram (http://bioinformatics.psb.ugent.be/webtools/Venn/). Further probe filtering for TF genes that were significantly induced by BPH or constitutively expressed in the resistant cultivar RHT was performed with the fold-change tool in Genespring GX 11.5.

\section{Quantitative reverse-transcription PCR (qPCR) analyses}

Purified RNA samples were treated with RNase-free DNase (NEB, Ipswich, USA) at $37^{\circ} \mathrm{C}$ for $1 \mathrm{~h}$. Reverse transcription was performed with the RevertAid ${ }^{\mathrm{mi}}$ First Strand cDNA Synthesis kit (Fermentas, Boston, USA) using Oligo $(\mathrm{dT})_{18}$ primers. The reactions were incubated at $42^{\circ} \mathrm{C}$ for $60 \mathrm{~min}$ and $70^{\circ} \mathrm{C}$ for $5 \mathrm{~min}$, chilled on ice for $5 \mathrm{~min}$, and the cDNA was stored at $-20^{\circ} \mathrm{C}$ until use. qPCR analyses were performed with Maxima $^{\text {TM }}$ SYBR Green qPCR Master Mix (2x) (Fermentas, Boston, USA) in $25-\mu$ l reaction mixtures following the manufacturer's instructions. The actin gene was used as an internal control to normalize Ct values obtained for each gene. Data analysis was performed according to the methods described by Livaka and Schmittgen [44]. Primer pairs are given in Online Resources (Additional file 1); these were designed using the PCR primer design tool primer3 (http://frodo.wi.mit.edu) according to the probe set target sequences accessible on the Affymetrix website http://www.affymetrix.com/analysis/index.affx).

\section{Results}

\section{RHT has an efficient resistant mechanism to brown}

\section{planthopper}

The Sri Lankan rice variety RHT contains a resistance gene, $B p h 3$, and shows resistance to all four biotypes of $\mathrm{BPH}$ [42]. This variety has an efficient resistance mechanism to BPH [42]. A previous study showed a low survival rate of BPH populations on RHT [1]. Another study on BPH showed that up to $80 \%$ of their time was spent on non-feeding behaviors, and they could not draw phloem sap from RHT [45]. When RHT and TN1 were exposed to BPH, RHT was less affected and survived longer than TN1. By 7 days of BPH infestation, TN1 plants were dead and dry, whereas RHT plants were still alive (Figure 1A). The herbivore host-plant 
choice experiment showed that BPH nymphs chose host plants randomly during the first $6-8 \mathrm{~h}$, but showed a preference for TN1 from $8 \mathrm{~h}$ after $\mathrm{BPH}$ infestation (Figure 1B). These findings indicated that RHT has an efficient resistance mechanism to $\mathrm{BPH}$.

\section{Transcription factor expression profiles in susceptible or resistant rice varieties}

We compared a series of Affymetrix rice genome arrays to measure the expression profiles of TF genes in two rice varieties before and after $\mathrm{BPH}$ infestation. According to the descriptions in the transcription factors database [43], we extracted a total of 2720 probes from the array that were annotated as TFs. In this study, the probes revealed 2038 TF genes $(74.9 \%)$ that were expressed at detectable levels after normalization. These could be subdivided into 79 gene families (Additional file 2). All of these TF genes were analyzed by pair-wise comparisons between resistant and susceptible varieties before and after $\mathrm{BPH}$ infestation using Genespring GX 11.5 software (Agilent Technologies). After comparison, of the 2038 TF genes with detectable expressions, 442 were identified as being related to $\mathrm{BPH}$-induced resistance and 229 TF genes were related to constitutive resistance in RHT (fold change $>2, P<0.05$ ) (Additional files $3 \& 4$ ). Those TF genes that were related to induced resistance were up-regulated or down-regulated by $\mathrm{BPH}$ attack. Those that were related to constitutive resistance were up-regulated or down-regulated in RHT compared with their respective expressions in $\mathrm{TN} 1$ at the same time points. These genes are considered as being specific to the resistant rice variety, RHT.

\section{Potential transcription factor genes associate with $\mathrm{BPH}-$ induced resistance}

As described above, there were 442 genes that were probably related to $\mathrm{BPH}$-induced resistance. These showed differential expressions between BPH-infested and noninfested plants. Further analysis showed that in the resistant rice variety RHT, 28 TF genes were specifically up-regulated and 39 genes were specifically downregulated at $8 \mathrm{~h}$ after $\mathrm{BPH}$ infestation (Figure $2 \mathrm{~A}$ ); at $24 \mathrm{~h}$ after BPH infestation, 31 genes were specifically upregulated and 20 genes were specifically down-regulated

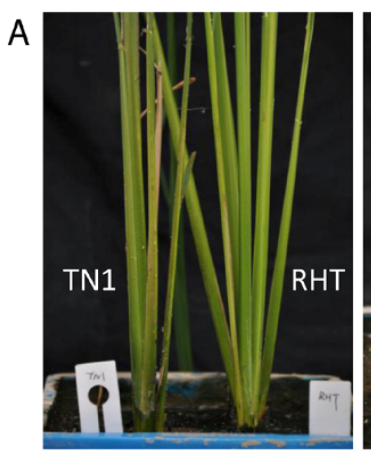

$1^{\text {st }}$ Day

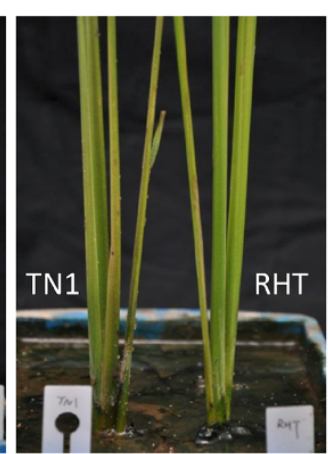

$3^{\text {rd }}$ Day

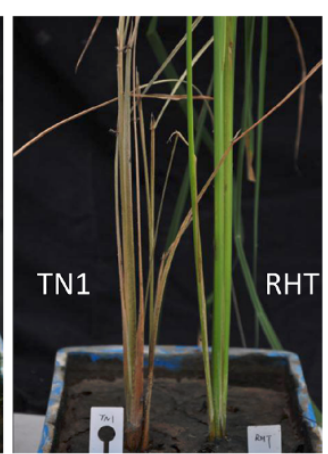

$7^{\text {th }}$ Day

B

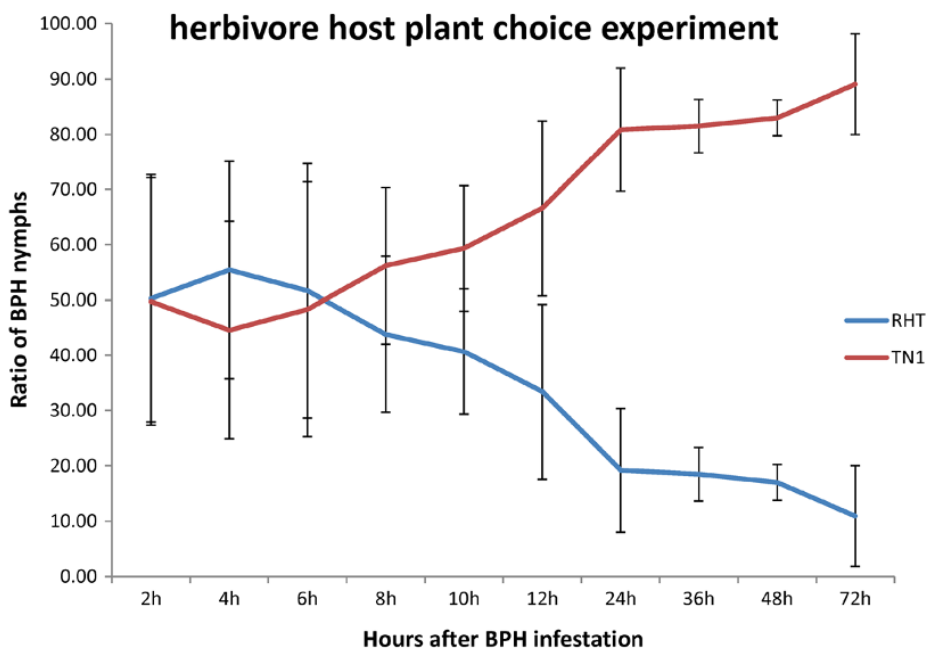

Figure 1 Brown planthopper (BPH) feeding and selection test on rice varieties RHT (resistant) and TN1 (susceptible). A, Phenotypes of RHT and TN1 on days 1, 3, and 7 of BPH infestation. B, BPH host selection experiment between rice varieties RHT and TN1 (72 h). 
(Figure 2B). However, at the same time points, there were 2-3 times more TF genes that were up- or downregulated by $\mathrm{BPH}$ attack in the susceptible rice variety TN1 (Figures 2A \& 2B).

As shown in Figures 2A and 2B, there were 105 upregulated TF genes in TN1 at $8 \mathrm{~h}$ after infestation and 154 at $24 \mathrm{~h}$ after infestation. There were 61 and 65 down-regulated genes at 8 and $24 \mathrm{~h}$ after infestation, respectively. In contrast, in the resistant variety RHT, there were more down-regulated than up-regulated genes after $\mathrm{BPH}$ infestation (Figures 2A).

The up- or down-regulated TF genes at two time points were further analyzed (Figures $2 \mathrm{C} \& 2 \mathrm{D}$ ). There were more TF genes up- or down-regulated in TN1 than in RHT after BPH attack. In other words, expressions of more TF genes were induced in the susceptible rice variety $\mathrm{TN} 1$ than in the resistant rice variety RHT. However, only a few genes were commonly expressed in the two rice varieties at different time points after $\mathrm{BPH}$ attack (Figures 2C \& 2D).

\section{Main families of TF genes related to $\mathrm{BPH}$ resistance}

A fold change of $>5$ was considered to indicate significantly differentially expressed TF genes. There were 119 TF genes related to induced resistance and 66 related to constitutive resistance with fold change values of $>5$
(Additional files $5 \& 6$ ). The 119 TF genes related to induced resistance belonged to $35 \mathrm{TF}$ gene families, mainly the WRKY, AP2-EREBP, HB, MYB, and NAC families (Figure 3A). The 66 TF genes related to constitutive resistance belonged to 25 families, mainly the WRKY, bHLH, MYB, and TRAF families (Figure 3B).

Further analyses indicated that there were significant differences in the expressions of zinc-finger TF family members between induced and constitutive TF genes. For instance, $\mathrm{C} 2 \mathrm{C} 2-\mathrm{CO}$ like and $\mathrm{C} 2 \mathrm{H} 2$ were the main zinc finger TF genes among the genes related to $\mathrm{BPH}$ induced resistance, whereas Tify and TRAF were the main zinc finger TF genes among those related to constitutive resistance (Figures $3 \mathrm{~A} \& 3 \mathrm{~B}$ ).

\section{Further analysis of TF genes related to $\mathrm{BPH}$-induced resistance}

To further identify TF genes that were induced by $\mathrm{BPH}$ and associated with $\mathrm{BPH}$ resistance, we screened 37 genes with $\mathrm{FC}$ values of $>10(P<0.05)$ (Table 1$)$. According to the $\mathrm{FC}$ value after $\mathrm{BPH}$ attack and the resistance of the two rice varieties to $\mathrm{BPH}$, the induced $\mathrm{TF}$ genes were divided into three categories: (1) Those that were strongly up- or down-regulated in $\mathrm{TN} 1$, but unaffected or barely affected in RHT $(\mathrm{FC}<5)$. These TF genes are likely to be sensitive to a biological stress, but are
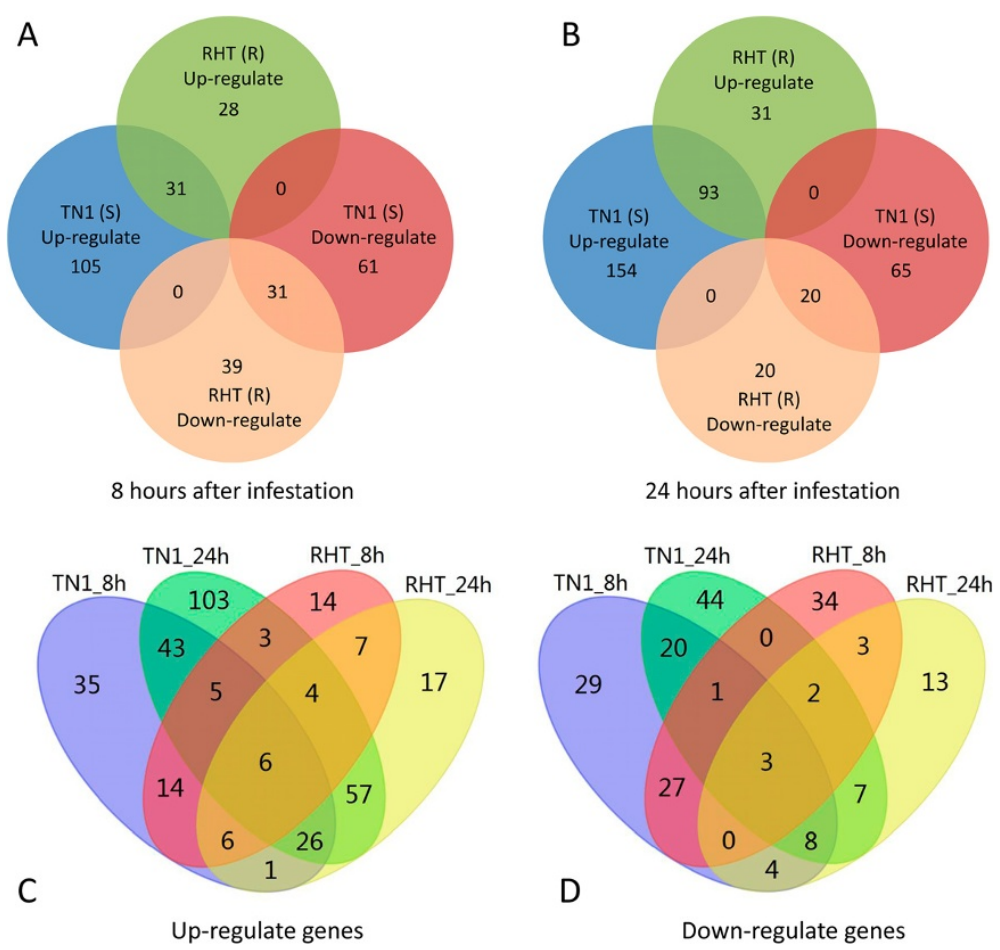

Figure 2 Analysis of 442 TF genes showing differential expression between resistant (RHT) and susceptible (TN1) rice varieties during BPH infestation. A, Up- or down-regulated TF genes in RHT or TN1 at $8 \mathrm{~h}$ after BPH infestation. B, Up- or down-regulated TF genes in RHT or TN1 at $24 \mathrm{~h}$ after BPH infestation. C, Up-regulated TF genes in RHT and TN1 at 8 and $24 \mathrm{~h}$ after BPH infestation. D, Down-regulated TF genes in RHT and TN1 at 8 and $24 \mathrm{~h}$ after BPH infestation. 


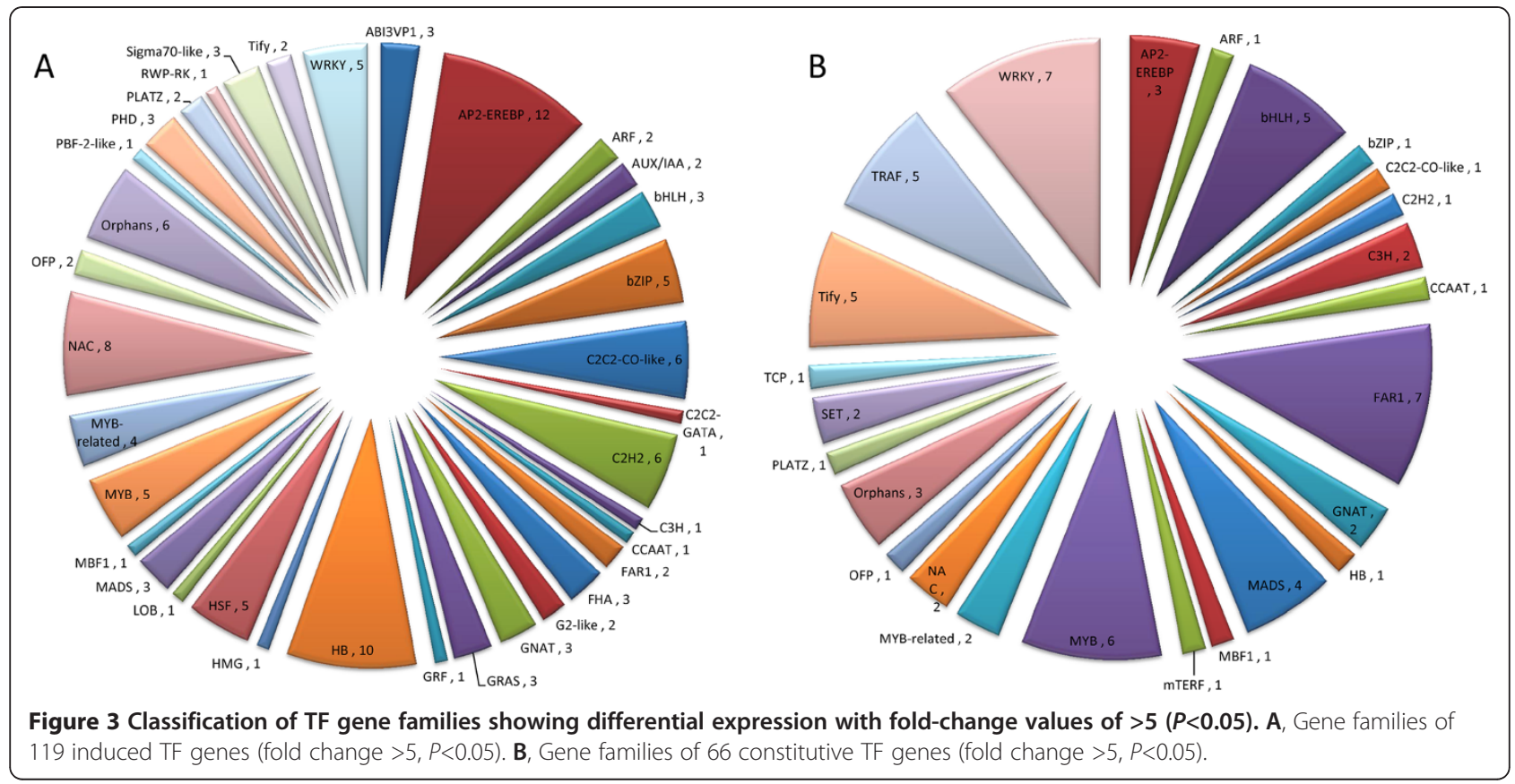

unlikely to participate in BPH-resistance (Table 1, lines 1-14). (2) Those that were strongly up- or downregulated at $8 \mathrm{~h}$ after $\mathrm{BPH}$ infestation in the two rice varieties, but showed expression levels similar to those before BPH attack thereafter. These genes probably participate in the early stages of the biological stress response (Table 1, lines 15-24). (3) Those that were up- or down-regulated in both rice varieties, or strongly downregulated only in RHT. These TF genes were likely to be involved in $\mathrm{BPH}$-inducible resistance, and included the remaining 13 TF genes (Table 1, lines 25-37).

\section{Transcription factor genes most likely related to constitutive resistance in $\mathrm{RHT}$}

To identify some specific TF genes from the resistant rice variety RHT, we analyzed TF gene expression in RHT uninfested or infested with BPH. In total, 26 genes had FC values of $>10(P<0.05)$ (Table 2$)$. These TF genes were divided into three categories: (1) Those that were strongly up- or down-regulated in RHT compared with TN1 before BPH infestation (13 genes, Table 2, lines 113). The expression patterns of these genes showed little difference before and after BPH infestation; therefore, these were genes that were constitutively expressed at high levels during the normal life cycle, and were not likely to be involved in the $\mathrm{BPH}$ resistance response. (2) Those that were strongly up- or down-regulated in RHT compared with TN1 before BPH attack (5 genes, Table 2, lines 14-19). The expression levels of these genes changed significantly after BPH attack. Therefore, these genes were probably involved in $\mathrm{BPH}$ resistance by regulating expression of resistance-related genes. (3) Those that showed almost the same expression levels in TN1 and RHT before BPH attack, but changed significantly in RHT after BPH attack. The remaining eight TF genes were in this category. These were the TF genes that most probably participated in BPH resistance in RHT (Table 2, lines 19-26).

\section{Confirmation of microarray expression profiles by qPCR}

To verify the expression profiles of TF genes, we selected six TF genes for qPCR analyses, and increased the number of analyzed time points (8 in total). The expression patterns of all six TF genes determined by qPCR were the same as those predicted from microarray analyses (Figures $4 \mathrm{~A}-\mathrm{F}$ ). Figures $4 \mathrm{~A}, 4 \mathrm{~B}$, and $4 \mathrm{C}$ show expressions of three $\mathrm{BPH}$-induced TF genes. Figure 4A shows expression of a Tify family TF gene; its expression was up-regulated in RHT and TN1 after BPH infestation. When this result is compared with data in Table 1 (line 13, showing expression at 8 and $24 \mathrm{~h}$ after $\mathrm{BPH}$ infestation), a slight difference was that its expression was up-regulated at $4 \mathrm{~h}$ after $\mathrm{BPH}$ infestation. Therefore, for accurate analysis of expressions of genes induced by $\mathrm{BPH}, \mathrm{qPCR}$ analyses provided additional data to array data. Figures $4 \mathrm{D}, 4 \mathrm{E}$, and $4 \mathrm{~F}$ illustrate expressions of three TF genes showing differential expression between uninfested and infested RHT. In the resistant variety RHT, these genes were up- or down-regulated before and after $\mathrm{BPH}$ infestation (Figure $4 \mathrm{D}-\mathrm{F}$, blue bars). However, in the susceptible rice variety $\mathrm{TN} 1$, their expressions were up-regulated after BPH attack (Figure 4 $\mathrm{D}-\mathrm{F}$, red bars). These TF genes represent those that are constitutively expressed in RHT in the normal life cycle, 
Table 1 TF genes related to BPH-induced resistance (fold change $>10 ; P<0.05$ )

\begin{tabular}{|c|c|c|c|c|c|c|c|c|c|c|}
\hline \multirow[t]{2}{*}{ No } & \multirow[t]{2}{*}{ Family } & \multirow[t]{2}{*}{ Gene ID } & \multicolumn{2}{|c|}{$\mathrm{T}_{8} / \mathrm{T}_{0}$} & \multicolumn{2}{|c|}{$\mathrm{T}_{24} / \mathrm{T}_{0}$} & \multicolumn{2}{|c|}{$\mathbf{R}_{8} / \mathbf{R}_{0}$} & \multicolumn{2}{|c|}{$\mathrm{R}_{24} / \mathrm{R}_{0}$} \\
\hline & & & $\mathrm{FC}$ & $\mathrm{RP}$ & $\mathrm{FC}$ & RP & $\mathrm{FC}$ & RP & FC & $\mathrm{RP}$ \\
\hline 1 & ABI3VP1 & Os03g0164300 & 10.14 & U & 10.28 & U & 1.50 & U & 4.31 & U \\
\hline 2 & AP2-EREBP & Os01g0224100 & 1.91 & $\mathrm{D}$ & 12.40 & D & 2.66 & D & 1.53 & $\mathrm{D}$ \\
\hline 3 & AP2-EREBP & Os03g0860100 & 19.22 & U & 8.30 & U & 2.13 & $U$ & 2.08 & U \\
\hline 4 & bZIP & Os03g0336200 & 4.37 & $U$ & 10.25 & U & 1.55 & $U$ & 3.08 & U \\
\hline 5 & C2C2-CO-like & Os09g0240200 & 29.47 & $\mathrm{D}$ & 1.22 & $\mathrm{D}$ & 3.57 & $\mathrm{D}$ & 1.83 & $U$ \\
\hline 6 & $\mathrm{C} 2 \mathrm{H} 2$ & Os03g0437200 & 10.14 & $U$ & 3.27 & U & 2.00 & $U$ & 1.67 & U \\
\hline 7 & FAR1 & Os08g0389800 & 7.46 & U & 11.87 & U & 1.36 & U & 1.36 & $\mathrm{D}$ \\
\hline 8 & FHA & Os12g0124000 & 14.24 & D & 1.23 & D & 3.91 & D & 1.38 & $\mathrm{D}$ \\
\hline 9 & GRAS & Os01g0948200 & 14.58 & $U$ & 6.82 & U & 1.30 & D & 2.28 & U \\
\hline 10 & $\mathrm{HB}$ & AK064665 & 8.88 & D & 14.77 & D & 2.18 & D & 3.32 & $\mathrm{D}$ \\
\hline 11 & NAC & Os02g0214500 & 10.52 & $U$ & 6.62 & U & 1.27 & D & 2.11 & D \\
\hline 12 & RWP-RK & Os09g0549450 & 2.69 & $\mathrm{D}$ & 10.01 & $\mathrm{D}$ & 1.39 & $\mathrm{D}$ & 2.83 & $\mathrm{D}$ \\
\hline 13 & Tify & Os03g0180800 & 10.45 & $U$ & 1.51 & U & 4.29 & $U$ & 3.75 & U \\
\hline 14 & WRKY & Os03g0335200 & 6.85 & U & 10.14 & U & 2.13 & U & 2.02 & U \\
\hline 15 & AP2-EREBP & Os07g0410700 & 5.35 & U & 1.73 & U & 10.02 & $U$ & 3.12 & U \\
\hline 16 & C2C2-CO-like & Os06g0298200 & 34.79 & U & 1.41 & U & 23.97 & $U$ & 1.02 & U \\
\hline 17 & C2C2-CO-like & Os09g0509700 & 11.88 & $U$ & 2.39 & $U$ & 5.49 & $U$ & 2.53 & U \\
\hline 18 & G2-like & Os01g0971800 & 1789.73 & $U$ & 1.71 & U & 804.09 & $U$ & 2.39 & U \\
\hline 19 & HSF & Os01g0733200 & 32.45 & U & 2.64 & U & 45.58 & U & 3.06 & U \\
\hline 20 & MBF1 & Os06g0592500 & 12.14 & $\mathrm{D}$ & 1.58 & D & 5.60 & D & 2.77 & D \\
\hline 21 & MYB-related & Os08g0157600 & 45.27 & D & 2.13 & U & 47.13 & $D$ & 1.70 & U \\
\hline 22 & Orphans & Os04g0493000 & 5.34 & D & 2.08 & U & 12.51 & $\mathrm{D}$ & 1.54 & U \\
\hline 23 & Sigma70-like & Os05g0586600 & 24.58 & D & 2.50 & $U$ & 92.84 & $\mathrm{D}$ & 1.87 & U \\
\hline 24 & Sigma70-like & Os05g0586600 & 38.02 & D & 2.82 & U & 53.11 & $\mathrm{D}$ & 1.50 & U \\
\hline 25 & GNAT & Os03g0205800 & 5.79 & U & 10.28 & $U$ & 5.43 & $U$ & 6.77 & U \\
\hline 26 & MADS & Os12g0501700 & 29.97 & U & 86.44 & U & 5.79 & U & 12.58 & U \\
\hline 27 & MYB-related & Os05g0579600 & 52.39 & D & 28.25 & D & 7.56 & $\mathrm{D}$ & 13.03 & $\mathrm{D}$ \\
\hline 28 & MYB-related & Os06g0728700 & 9.13 & D & 6.10 & U & 16.15 & $\mathrm{D}$ & 3.19 & U \\
\hline 29 & NAC & Os12g0477400 & 7.13 & U & 22.52 & U & 2.00 & U & 8.33 & U \\
\hline 30 & NAC & Os11g0126900 & 3.56 & $U$ & 11.59 & $U$ & 1.06 & $\mathrm{D}$ & 6.55 & U \\
\hline 31 & $\mathrm{HB}$ & Os03g0109400 & 5.61 & U & 17.60 & U & 3.09 & U & 7.98 & U \\
\hline 32 & HMG & Os02g0258200 & 1.02 & U & 7.29 & $\mathrm{D}$ & 14.23 & $D$ & 7.63 & D \\
\hline 33 & MYB-related & Os02g0685200 & 2.34 & D & 10.57 & U & 5.75 & $\mathrm{D}$ & 3.37 & U \\
\hline 34 & PHD & Os06g0187000 & 1.10 & U & 6.72 & $\mathrm{D}$ & 22.53 & $\mathrm{D}$ & 3.62 & $\mathrm{D}$ \\
\hline 35 & GRAS & Os06g0127800 & 1.14 & U & 3.59 & D & 30.11 & $\mathrm{D}$ & 7.77 & $\mathrm{D}$ \\
\hline 36 & GRF & Os02g0678800 & 1.39 & U & 1.75 & $\mathrm{D}$ & 11.22 & D & 2.60 & D \\
\hline 37 & $\mathrm{HB}$ & Os10g0575600 & 1.78 & U & 1.62 & $\mathrm{D}$ & 12.97 & $\mathrm{D}$ & 1.25 & $D$ \\
\hline
\end{tabular}

${ }^{*} T_{0}$ and $R_{0}$ refer to rice cultivars TN1 and RHT before attack by BPH $(0 h) ; T_{8}, T_{24}, R_{8}$, and $R_{24}$ refer to the same cultivars sampled 8 or $24 \mathrm{~h}$ after attack by BPH; FC, fold change; RP, regulation pattern; $U$, up-regulation; $D$, down-regulation. 
Table 2 TF genes that were unaffected by BPH and those that showed constitutive differential expression in RHT (FC>10, $P<0.05)$

\begin{tabular}{|c|c|c|c|c|c|c|c|c|}
\hline \multirow[t]{2}{*}{ No } & \multirow[t]{2}{*}{ Family } & \multirow[t]{2}{*}{ Gene ID } & \multicolumn{2}{|c|}{$\mathrm{R}_{0} / \mathrm{T}_{0}$} & \multicolumn{2}{|c|}{$\mathrm{R}_{8} / \mathrm{T}_{8}$} & \multicolumn{2}{|c|}{$\mathrm{R}_{24} / \mathrm{T}_{24}$} \\
\hline & & & $\overline{F C}$ & $\overline{\mathrm{RP}}$ & $\overline{\mathrm{FC}}$ & $\mathrm{RP}$ & $\overline{F C}$ & RP \\
\hline 1 & bHLH & Os01g0108400 & 60.02 & $U$ & 30.02 & U & 59.83 & $U$ \\
\hline 2 & MYB-related & Os07g0634900 & 584.50 & U & 1219.35 & U & 216.66 & $U$ \\
\hline 3 & $\mathrm{C} 3 \mathrm{H}$ & Os01g0192000 & 24.32 & U & 25.73 & U & 25.62 & U \\
\hline 4 & FAR1 & Os05g0128700 & 20.44 & D & 106.39 & D & 29.94 & $D$ \\
\hline 5 & FAR1 & Os04g0316800 & 10.96 & D & 12.35 & D & 17.78 & D \\
\hline 6 & GNAT & Os08g0260000 & 9.35 & D & 9.66 & D & 12.28 & $D$ \\
\hline 7 & MADS & Os08g0112700 & 9.61 & U & 37.64 & U & 23.08 & U \\
\hline 8 & MADS & Os04g0387500 & 12.88 & D & 41.15 & D & 13.56 & $D$ \\
\hline 9 & mTERF & Os06g0224400 & 7.50 & D & 6.11 & D & 23.84 & $D$ \\
\hline 10 & GNAT & Os02g0806000 & 14.71 & U & 21.75 & U & 19.65 & U \\
\hline 11 & Orphans & Os01g0530300 & 6.53 & U & 5.20 & U & 50.91 & $U$ \\
\hline 12 & TRAF & Os03g0667100 & 223.89 & D & 118.10 & D & 110.88 & D \\
\hline 13 & TRAF & Os08g0227200 & 21.53 & U & 33.62 & $U$ & 17.75 & $u$ \\
\hline 14 & bHLH & Os04g0301500 & 5.53 & $D$ & 13.20 & D & 2.85 & D \\
\hline 15 & bZIP & Os01g0859500 & 10.39 & $\mathrm{D}$ & 6.31 & $\mathrm{D}$ & 1.17 & U \\
\hline 16 & FAR1 & Os08g0389800 & 25.22 & U & 4.59 & U & 1.56 & $u$ \\
\hline 17 & FAR1 & Os06g0246700 & 10.18 & D & 1.50 & U & 1.35 & $u$ \\
\hline 18 & Orphans & LOC_Os04g13480.1 & 25.93 & U & 1.16 & D & 3.73 & U \\
\hline 19 & Tify & Os09g0439200 & 4.82 & U & 7.20 & U & 30.32 & $u$ \\
\hline 20 & TRAF & LOC_Os08g41240.1 & 4.41 & D & 3.97 & D & 10.30 & D \\
\hline 21 & MYB & Os02g0624300 & 2.17 & D & 11.48 & D & 4.38 & D \\
\hline 22 & ARF & Os04g0664400 & 1.99 & U & 14.99 & D & 4.05 & $u$ \\
\hline 23 & NAC & Os01g0339500 & 1.70 & D & 1.64 & U & 10.83 & $\mathrm{D}$ \\
\hline 24 & SET & Os08g0244400 & 1.92 & U & 18.66 & U & 8.11 & U \\
\hline 25 & TCP & Os03g0706500 & 1.36 & D & 11.25 & D & 2.18 & $U$ \\
\hline 26 & Tify & Os04g0395800 & 1.10 & $U$ & 14.08 & D & 1.20 & D \\
\hline
\end{tabular}

${ }^{*} T_{0}$ and $R_{0}$ refer to rice cultivars TN1 and RHT before attack by BPH $(0 h) ; T_{8}, T_{24}, R_{8}$, and $R_{24}$ refer to the same cultivars sampled 8 or $24 \mathrm{~h}$ after attack by BPH; FC, fold change; RP, regulation pattern; $U$, up-regulation; $D$, down-regulation.

and most are unlikely to be involved in the $\mathrm{BPH}$ resistance reaction.

\section{Discussion}

Studies on the resistance mechanism of rice to $\mathrm{BPH}$ have been based on physical interactions, and more recently, on gene expression levels [9,11,46-48]. There is increasing evidence that TF genes play important roles in plant defense responses against phloem-feeding insects. TF genes represent a key element in the modification of gene expression during the plant defense reaction $[8,17,18,49]$.

Here, we provide an overview of the expression profile of TF genes related to $\mathrm{BPH}$ resistance, based on comparisons of susceptible and resistant rice plants. Using TF gene probes, we detected 2038 TF genes showing differential expressions between the two rice varieties RHT and TN1 (Additional file 2). Of these, 442 TF genes were probably related to $\mathrm{BPH}$-induced resistance, because their expressions were increased or decreased in response to BPH infestation (Additional file 3). All these induced TF genes showed an interesting expression pattern at two time points ( 8 or $24 \mathrm{~h}$ ) after $\mathrm{BPH}$ infestation (Figure 2A, 2B). After BPH feeding, the susceptible strain TN1 had more up- or down-regulated TF genes than did the resistant strain RHT. According to the different patterns of gene expression profiles, more TF genes were induced by $\mathrm{BPH}$ in the susceptible rice variety TN1 than in the resistant rice variety RHT. For instance, as shown in Figure 2A and 2B, TF genes specially up-regulated at 8 , and 24 hours in TN1 were 105 and 154, respectively; while the numbers in RHT were 28 and 31 . And TF genes specially down-regulated at 8 , and 24 hours in TN1 were 61 and 65, respectively; while the 


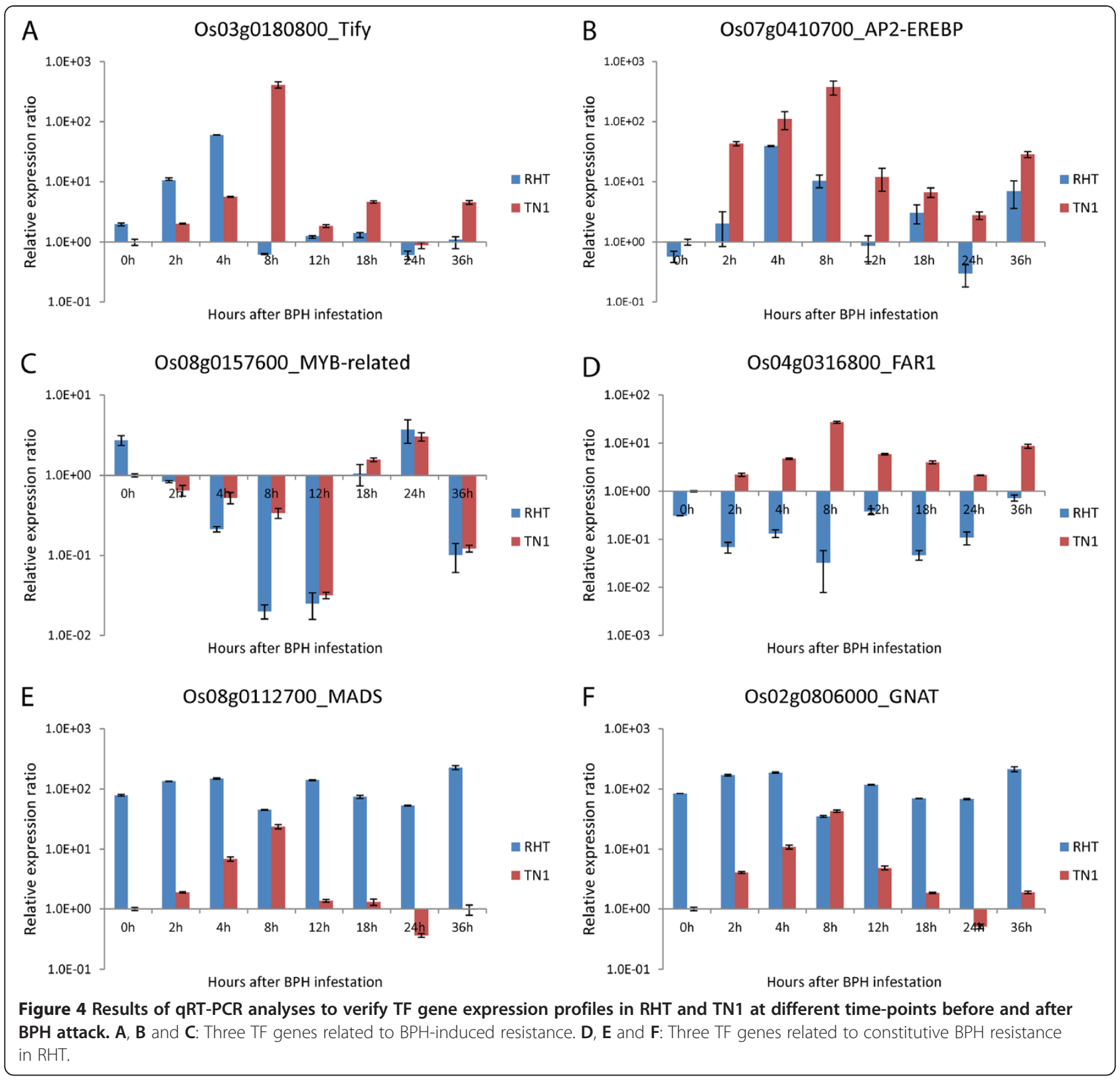

numbers in RHT were 39 and 20. Because induced resistance is defined from the point of view of the herbivore and it does not necessarily benefit the plant. This means that the induced resistance may render the plant more susceptible to other stresses [4]. This may be the one important reason why TN1 rice is more susceptible than RHT. Further analysis results also support this point of view. In TN1, there were more up-regulated than down-regulated TF genes in response to $\mathrm{BPH}$. In contrast, in RHT, there were more down-regulated than up-regulated genes in response to $\mathrm{BPH}$ (Figures 2A). This finding suggested that the damage caused by $\mathrm{BPH}$ feeding to the susceptible variety $\mathrm{TN} 1$ triggered expressions of a series of genes. The products of these genes may play roles in repairing damage to the phloem to prevent the loss of phloem sap, and in defense against invasion of the pathogen and bacteria. In the resistant variety RHT, this reaction involved cessation of several metabolic pathways to prevent the loss of phloem sap, and metabolic activity was repressed throughout the entire plant. This is an efficient method of defense against $\mathrm{BPH}$ feeding, because it reduces the amount of substances supplied to the phloem, similar to the function of the resistance gene Bph14 [49]. Therefore, various TFs could have different functions in this reaction. This finding provided evidence at the molecular level for why 
the resistant cultivar, RHT, suffered less damage than the susceptible cultivar, TN1.

Studies on the physical test phenotype by EPG (electrical penetration graph) of these cultivars showed that TN1 had a greater damage score after exposure to $\mathrm{BPH}$, while the phloem sap of RHT could not be extracted constantly by $\mathrm{BPH}$ stylets $[1,45]$. According to this study, the expressions of many TF genes were induced in TN1 by exposure to $\mathrm{BPH}$. This would probably lead to metabolic disorders in $\mathrm{TN} 1$, which probably the reason why this rice variety is susceptible to BPH. However, in RHT, far fewer TF genes were induced after BPH exposure. Most of the TF genes showing altered expressions in RHT were down-regulated (Figure 2A and 2B), so we boldly deduced that certain metabolic pathways were turned off. This may explain why BPH was unable to extract nutrition from the RHT phloem sap.

Among the down-regulated TF genes, all members of FHA, Orphans, and Sigma70-like families, and most members of the MYB family were significantly downregulated at $8 \mathrm{~h}$ after BPH infestation (Additional file 5). A similar pattern of regulation also occurred in Arabidopsis after aphid infestation [17]. Members of the MYB family have highly diversified biological functions, and the expressions of most Arabidopsis MYB genes were responsive to one or multiple types of hormone and stress treatments [50]. Studies of the Arabidopsis defense response to chewing insects showed that knockdown of a MYB gene, AtMYB102, enhanced susceptibility to white cabbage butterfly (Pieris rapae) [51]. In tobacco, transgenic plants overexpressing a wheat MYB gene, TaPIMP1, showed significantly enhanced resistance to the pathogen Ralstonia solanacearum, and increased tolerance to drought and salt stresses [30]. Another study on TFs suggested that several members of the MYB family play important roles in photosynthesis and metabolism [52]. During their physiological responses to insects, plants reallocate energy from photosynthesis to the defense response [9]. During BPH feeding, the chlorophyll level, net photosynthetic rate, stomatal conductance, and transpiration rates significantly decreased in the susceptible cultivar, MH63 [11]. These patterns of decreased metabolic activity were consistent with the down-regulation of MYB family genes observed in BPHinfested rice plants in this study. The down-regulation of these genes may have played a role in reducing photosynthesis. Microarray analyses have shown that downregulation of TF genes occurs during compatible plantaphid interactions [53].

In both rice varieties used in the present study, the up-regulated TF genes after 8-h of BPH infestation included most members of the AP2-EREBP, NAC, and WRKY families and all members of the ABI3VP1 family (Additional file 5). The AP2 TF family is one of several that are unique to the plant lineage. This family, whose members contain an EREBP (ethylene responsive element binding factor) domain, is involved in regulation of plant disease resistance [19]. Genes encoding members of the AP2-EREBP family of TFs are also involved in plant resistance to insects. The AP2-EREBP TF response to herbivore attack might depend on a hormone-dependent signaling pathway. Over-expression of OsERF3 positively regulated TrypPI activity and boosted the resistance of rice to the chewing herbivore $\mathrm{SSB}$, whereas it negatively regulated resistance against $\mathrm{BPH}$ [8]. The expression pattern of up-regulated AP2EREBP genes may reflect those induced by physical damage during $\mathrm{BPH}$ feeding, and the down-regulated AP2 TFs may be involved in the BPH resistance interaction. Previous studies on NAC, WRKY, and Zn-finger TF families mainly focused on responses to fungal and bacterial pathogens $[23,25,33]$. According to those studies, their functions rely on JA and SA signaling pathways, which are involved in the BPH-resistance response.

We narrowed down the number of TF genes that were probably involved in $\mathrm{BPH}$ resistance by increasing the FC value to $>10(P<0.05)$. At this FC level, there were 37 TF genes that were $\mathrm{BPH}$-induced and 26 associated with constitutive resistance in RHT. Further analysis indicated that there were 13 and 8 TF genes most probably involved in $\mathrm{BPH}$-induced and constitutive resistance, respectively. Further research is underway to study these 21 genes in more detail. Most of the microarray expression profiles were consistent with the qPCR results. (Figure 4 \& Additional file 7). However, only a few time points were analyzed in the microarray analyses, and so it is difficult to determine the exact time at which gene expression peaked after induction by $\mathrm{BPH}$. To address this point, more time points should be included in qPCR analyses of gene expression.

\section{Conclusions}

TFs are up-stream regulators that control transcription by DNA binding. By analyzing the TF gene expression profiles after BPH feeding, especially those of genes that were differentially expressed between resistant (RHT) and susceptible (TN1) rice varieties, we obtained information about the resistance mechanism to $\mathrm{BPH}$ at the molecular level. Our results indicate that the levels of gene expression play an important role in the plant defense reaction. The down-regulation of $\mathrm{TF}$ genes will repress many active pathways, which can prevent further damage related to loss of water and nutrients.

An interesting aspect of the molecular mechanism of TFs in plant resistance to BPH was that the TF genes showing down-regulated or repressed expressions were probably the main reason for BPH resistance in RHT. 
We identified a total of 21 TF genes that are probably involved in BPH resistance in the resistant rice variety, RHT.

\section{Additional files}

\author{
Additional file 1: Primer pairs for quantitative RT-PCR to verify \\ relative expression levels of transcription factor genes. \\ Additional file 2: All transcription factor genes detected by \\ microarray analyses. This file lists all 2038 transcription factor genes \\ detected by microarray analyses, and shows TF genes showing \\ differential expression profiles between two rice varieties TN1 and RHT \\ before and after attack by BPH.
}

Additional file 3: Transcription factor genes related to $\mathrm{BPH}$-induced resistance showing changes in expression with fold-change values of $>\mathbf{2}$ after BPH infestation $(\boldsymbol{P}<\mathbf{0 . 0 5})$. This file lists 442 transcription factor genes showing changes in expression after $\mathrm{BPH}$ attack (foldchange $>2, P<0.05$ ). It also shows TF genes showing differential expression profiles between two rice varieties TN1 and RHT before and after attack by BPH.

Additional file 4: Transcription factor genes related to constitutive resistance in RHT showing changes in expression with fold-change values of $>\mathbf{2}(\boldsymbol{P}<\mathbf{0 . 0 5})$. This file lists 229 transcription factor genes that were up-regulated or down-regulated before and after BPH attack in RHT compared with their respective expressions in TN1.

Additional file 5: BPH-induced transcription factor genes showing altered expression after BPH infestation with fold-change values of $>\mathbf{5}(\boldsymbol{P}<\mathbf{0 . 0 5})$. This file lists 119 transcription factor genes showing altered expression after $\mathrm{BPH}$ attack with fold-change values of $>5(P<0.05)$. It also shows TF genes showing differential expression profiles between two rice varieties of TN1 and RHT before and after attack by BPH.

Additional file 6: Transcription factor genes related to constitutive resistance in RHT with fold-change values of $>5(P<0.05)$. This file lists 66 transcription factor genes showing up- or down-regulated expressions before and after BPH attack in RHT compared with their respective expressions in TN1.

Additional file 7: The other results of qRT-PCR analyses to verify TF gene expression profiles in RHT and TN1 at different time-points before and after BPH attack.

\section{Abbreviations}

BPH: Brown planthopper; TF: Transcription factors; RHT: Rathu Heenati; TN1: Taichun Native 1; FC: Fold change; HR: Hypersensitive response; SA: Salicylic acid; JA: Jasmonic acid; ET: Ethylene; WBPH: White-backed planthopper; qPCR: Quantitative reverse-transcription PCR.

\section{Competing interests}

The authors declare that they have no competing interests.

\section{Authors' contributions}

YW, XM conceived and designed the study, and wrote the manuscript. YW and $\mathrm{HL}$ analyzed the experiment results, prepared figures and tables, and revised the manuscript. $\mathrm{HG}$ and $\mathrm{HZ}$ collected samples and conducted qRTPCR analyses. All authors read and approved the final manuscript.

\section{Acknowledgements}

This work was supported by the National Basic Research Program of China (2010CB126202), the National Transgenic Great Subject from the Ministry of Agriculture of China (2009ZX08001-003B), and grant (10JC1416300 and RCT2008001) from the Shanghai and Xuhui Commission of Science.

\section{Author details}

${ }^{1}$ Key Laboratory of Insect Developmental and Evolutionary Biology, Institute of Plant Physiology and Ecology, Shanghai Institutes for Biological Sciences, Chinese Academy of Sciences, Shanghai 200032, P.R. China. ${ }^{2}$ Graduate School of Chinese Academy of Sciences, Shanghai 200032, P.R. China.
Received: 14 June 2012 Accepted: 5 December 2012

Published: 10 December 2012

\section{References}

1. Seo BY, Kwon Y-H, Jung JK, Kim G-H: Electrical penetration graphic waveforms in relation to the actual positions of the stylet tips of Nilaparvata lugens in rice tissue. J Asia Pac Entomol 2009, 12(2):89-95.

2. Heinrichs EA: Control of leafhopper and planthopper vectors of rice viruses. In Leafhopper vectors and plant disease agents. Edited by Maramorosch K, Harris KF. New York: Academic Press; 1979:529-560.

3. Normile D: Agricultural research. Reinventing rice to feed the world. Science 2008, 321(5887):330-333.

4. Gregg AH, Andreas S: Direct Defenses in Plants and Their Induction by Wounding and Insect Herbivores. In Induced Plant Resistance to Herbivory, Volume 1. 1st edition. Edited by Andreas S. Stuttgart, Germany: Springer; 2008:7-30

5. Underwood N, Rausher M: Comparing the consequences of induced and constitutive plant resistance for herbivore population dynamics. Am Nat 2002, 160(1):20-30.

6. Zander M, La Camera S, Lamotte O, Metraux JP, Gatz C: Arabidopsis thaliana class-II TGA transcription factors are essential activators of jasmonic acid/ethylene-induced defense responses. Plant J 2010, 61(2):200-210

7. Zarate SI, Kempema LA, Walling LL: Silverleaf whitefly induces salicylic acid defenses and suppresses effectual jasmonic acid defenses. Plant Physiol 2007, 143(2):866-875.

8. Lu J, Ju H, Zhou G, Zhu C, Erb M, Wang X, Wang P, Lou Y: An EAR-motif-containing ERF Transcription Factor Affects Herbivore-Induced Signaling, Defense and Resistance in Rice. Plant J 2011, 68(4):583-596.

9. Yuan $H$, Chen $X$, Zhu L, He G: Identification of genes responsive to brown planthopper Nilaparvata lugens Stal (Homoptera: Delphacidae) feeding in rice. Planta 2005, 221(1):105-112.

10. Walling LL: The Myriad Plant Responses to Herbivores. J Plant Growth Regul 2000, 19(2):195-216.

11. Wang $Y$, Wang $X$, Yuan $H$, Chen $R$, Zhu L, He R, He G: Responses of two contrasting genotypes of rice to brown planthopper. Mol Plant Microbe Interact 2008, 21(1):122-132.

12. Zhang F, Zhu L, He G: Differential gene expression in response to brown planthopper feeding in rice. J Plant Physiol 2004, 161(1):53-62.

13. Wang Y, Li H, Si Y, Zhang H, Guo H, Miao X: Microarray analysis of broadspectrum resistance derived from an indica cultivar Rathu Heenati. Planta 2012, 235(4):829-840.

14. Latchman DS: Transcription factors: an overview. Int J Biochem Cell Biol 1997, 29(12):1305-1312.

15. Karin M: Too many transcription factors: positive and negative interactions. New Biol 1990, 2(2):126-131.

16. Riechmann JL, Ratcliffe OJ: A genomic perspective on plant transcription factors. Curr Opin Plant Biol 2000, 3(5):423-434

17. Gao LL, Kamphuis LG, Kakar K, Edwards OR, Udvardi MK, Singh KB: Identification of potential early regulators of aphid resistance in Medicago truncatula via transcription factor expression profiling. New Phytol 2010, 186(4):980-994.

18. Libault M, Wan J, Czechowski T, Udvardi M, Stacey G: Identification of 118 Arabidopsis transcription factor and 30 ubiquitin-ligase genes responding to chitin, a plant-defense elicitor. Mol Plant Microbe Interact 2007, 20(8):900-911.

19. Gutterson N, Reuber TL: Regulation of disease resistance pathways by AP2/ERF transcription factors. Curr Opin Plant Biol 2004, 7(4):465-471.

20. Yang B, Jiang Y, Rahman MH, Deyholos MK, Kav NN: Identification and expression analysis of WRKY transcription factor genes in canola (Brassica napus L.) in response to fungal pathogens and hormone treatments. BMC Plant Biol 2009, 9:68.

21. Ren CM, Zhu Q, Gao BD, Ke SY, Yu WC, Xie DX, Peng W: Transcription factor WRKY70 displays important but no indispensable roles in jasmonate and salicylic acid signaling. J Integr Plant Biol 2008, 50(5):630-637.

22. Ulker B, Shahid Mukhtar M, Somssich IE: The WRKY70 transcription factor of Arabidopsis influences both the plant senescence and defense signaling pathways. Planta 2007, 226(1):125-137. 
23. Knoth C, Ringler J, Dangl $J$, Eulgem T: Arabidopsis WRKY70 is required for full RPP4-mediated disease resistance and basal defense against Hyaloperonospora parasitica. Mol Plant Microbe Interact 2007, 20(2):120-128.

24. Wang H, Hao J, Chen $X$, Hao Z, Wang X, Lou Y, Peng Y, Guo Z: Overexpression of rice WRKY89 enhances ultraviolet B tolerance and disease resistance in rice plants. Plant Mol Biol 2007, 65(6):799-815.

25. Deng H, Liu H, Li X, Xiao J, Wang S: A CCCH-Type Zinc Finger Nucleic Acid-Binding Protein Quantitatively Confers Resistance against Rice Bacterial Blight Disease. Plant Physiol 2012, 158:876-889.

26. Sun SJ, Guo SQ, Yang X, Bao YM, Tang HJ, Sun H, Huang J, Zhang HS: Functional analysis of a novel Cys2/His2-type zinc finger protein involved in salt tolerance in rice. J Exp Bot 2010, 61(10):2807-2818.

27. Zhou Z, Li G, Lin C, He C: Conidiophore stalk-less1 encodes a putative zinc-finger protein involved in the early stage of conidiation and mycelial infection in Magnaporthe oryzae. Mol Plant Microbe Interact 2009, 22(4):402-410

28. Huang J, Yang $X$, Wang MM, Tang HJ, Ding LY, Shen Y, Zhang HS: A novel rice $\mathrm{C} 2 \mathrm{H} 2$-type zinc finger protein lacking DLN-box/EAR-motif plays a role in salt tolerance. Biochimica Et Biophysica Acta 2007, 1769(4):220-227.

29. He Y, Li W, LV J, Jia Y, Wang M, Xia G: Ectopic expression of a wheat MYB transcription factor gene, TaMYB73, improves salinity stress tolerance in Arabidopsis thaliana. J Exp Bot 2011, 63(3):1511-1522.

30. Liu H, Zhou X, Dong N, Liu X, Zhang H, Zhang Z: Expression of a wheat MYB gene in transgenic tobacco enhances resistance to Ralstonia solanacearum, and to drought and salt stresses. Funct Integr Genomics 2011, 11(3):431-443

31. Sun H, Huang $X, X u X$, Lan H, Huang J, Zhang HS: ENAC1, a NAC Transcription Factor, is an Early and Transient Response Regulator Induced by Abiotic Stress in Rice (Oryza sativa L.). Mol Biotechnol 2011, 52(2):101-110

32. Takasaki H, Maruyama K, Kidokoro S, Ito Y, Fujita Y, Shinozaki K, YamaguchiShinozaki K, Nakashima K: The abiotic stress-responsive NAC-type transcription factor OsNAC5 regulates stress-inducible genes and stress tolerance in rice. Mol Genet Genomics 2010, 284(3):173-183.

33. Xia N, Zhang G, Liu XY, Deng L, Cai GL, Zhang Y, Wang XJ, Zhao J, Huang $L L$, Kang ZS: Characterization of a novel wheat NAC transcription factor gene involved in defense response against stripe rust pathogen infection and abiotic stresses. Mol Biol Rep 2010, 37(8):3703-3712.

34. Wang X, Basnayake BM, Zhang H, Li G, Li W, Virk N, Mengiste T, Song F: The Arabidopsis ATAF1, a NAC transcription factor, is a negative regulator of defense responses against necrotrophic fungal and bacterial pathogens. Mol Plant Microbe Interact 2009, 22(10):1227-1238.

35. Liu C, Wu Y, Wang X: bZIP transcription factor OsbZIP52/RISBZ5: a potential negative regulator of cold and drought stress response in rice. Planta 2012, 235(6):1159-1169.

36. Guo M, Chen Y, Du Y, Dong Y, Guo W, Zhai S, Zhang H, Dong S, Zhang Z, Wang $Y$, et al: The bZIP transcription factor MoAP1 mediates the oxidative stress response and is critical for pathogenicity of the rice blast fungus Magnaporthe oryzae. PLoS Pathog 2011, 7(2):e1001302.

37. Liao Y, Zou HF, Wei W, Hao YJ, Tian AG, Huang J, Liu YF, Zhang JS, Chen SY: Soybean GmbZIP44, GmbZIP62 and GmbZIP78 genes function as negative regulator of $A B A$ signaling and confer salt and freezing tolerance in transgenic Arabidopsis. Planta 2008, 228(2):225-240.

38. Lee SC, Choi HW, Hwang IS, du Choi S, Hwang BK: Functional roles of the pepper pathogen-induced bZIP transcription factor, CAbZIP1, in enhanced resistance to pathogen infection and environmental stresses. Planta 2006, 224(5):1209-1225.

39. Zhao X, Huang J, Yu H, Wang L, Xie W: Genomic survey, characterization and expression profile analysis of the peptide transporter family in rice (Oryza sativa L.). BMC Plant Biol 2010, 10:92.

40. Zhang H, Peng H, Li P, Deng Q, Xu P, Li Y, Wang X, Wu X: The microarray analysis for gene expression in haploids and diploids derived from twin-seedling rice. Sci China C Life Sci 2008, 51(6):503-512.

41. Yamakawa H, Hirose T, Kuroda M, Yamaguchi T: Comprehensive expression profiling of rice grain filling-related genes under high temperature using DNA microarray. Plant Physiol 2007, 144(1):258-277.

42. Lakshminarayana A, Khush GS: New genes for resistance to the brown planthopper in rice. Crop Sci 1977, 17(1):96-100

43. Perez-Rodriguez P, Riano-Pachon DM, Correa LG, Rensing SA, Kersten B, Mueller-Roeber B: PInTFDB: updated content and new features of the plant transcription factor database. Nucleic Acids Res 2010, 38(Database issue):D822-D827.

44. Livak K, Schmittgen D: Analysis of Relative Gene Expression Data Using Real-Time Quantitative PCR and the 2-CT Method. Methods 2001, 25:402-481

45. Ghaffar MB, Pritchard J, Ford-Lloyd B: Brown planthopper (N. lugens Stal) feeding behaviour on rice germplasm as an indicator of resistance. PLoS One 2011, 6(7):e22137.

46. Kojima Y, Sasaki S, Hayashi Y, Tsujimoto G, Kohri K: Subtypes of alpha1-adrenoceptors in $\mathrm{BPH}$ : future prospects for personalized medicine. Nat Clin Pract Urol 2009, 6(1):44-53.

47. Park DS, Lee SK, Lee JH, Song MY, Song SY, Kwak DY, Yeo US, Jeon NS, Park $S K, Y i$, et al: The identification of candidate rice genes that confer resistance to the brown planthopper (Nilaparvata lugens) through representational difference analysis. Theor Appl Genet 2007, 115(4):537-547.

48. Wei Z, Hu W, Lin Q, Cheng X, Tong M, Zhu L, Chen R, He G: Understanding rice plant resistance to the Brown Planthopper (Nilaparvata lugens): a proteomic approach. Proteomics 2009, 9(10):2798-2808.

49. Du B, Zhang W, Liu B, Hu J, Wei Z, Shi Z, He R, Zhu L, Chen R, Han B, et al: Identification and characterization of Bph14, a gene conferring resistance to brown planthopper in rice. Proc Natl Acad Sci USA 2009, 106(52):22163-22168.

50. Yanhui C, Xiaoyuan Y, Kun H, Meihua L, Jigang L, Zhaofeng G, Zhiqiang L, Yunfei Z, Xiaoxiao W, Xiaoming Q, et al: The MYB transcription factor superfamily of Arabidopsis: expression analysis and phylogenetic comparison with the rice MYB family. Plant Mol Biol 2006, 60(1):107-124.

51. De Vos M, Denekamp M, Dicke M, Vuylsteke M, Van Loon L, Smeekens SC, Pieterse CM: The Arabidopsis thaliana Transcription Factor AtMYB102 Functions in Defense Against the Insect Herbivore Pieris rapae. Plant Signal Behav 2006, 1(6):305-311.

52. Saibo NJ, Lourenco T, Oliveira MM: Transcription factors and regulation of photosynthetic and related metabolism under environmental stresses. Ann Bot 2009, 103(4):609-623.

53. Macedo TB, Bastos CS, Higley LG, Ostlie KR, Madhavan S: Photosynthetic responses of soybean to soybean aphid (Homoptera: Aphididae) injury. J Econ Entomol 2003, 96(1):188-193.

doi:10.1186/1471-2164-13-687

Cite this article as: Wang et al:: Identification of transcription factors potential related to brown planthopper resistance in rice via microarray expression profiling. BMC Genomics 2012 13:687.

\section{Submit your next manuscript to BioMed Central and take full advantage of:}

- Convenient online submission

- Thorough peer review

- No space constraints or color figure charges

- Immediate publication on acceptance

- Inclusion in PubMed, CAS, Scopus and Google Scholar

- Research which is freely available for redistribution
C) Biomed Central 